\title{
Diagnóstico y tratamiento de las complicaciones urológicas del trasplante renal
}

\author{
Gonzalo Rodríguez V, Rivero Martínez MD, Trueba Arguiñarena J, Calleja Escudero J, \\ Müller Arteaga C, Fernández del Busto E. \\ Hospital Clínico Universitario de Valladolid. \\ Actas Urol Esp 2006; 30 (6): 619-625
}

\section{RESUMEN}

DIAGNÓSTICO Y TRATAMIENTO DE LAS COMPLICACIONES UROLÓGICAS DEL TRASPLANTE RENAL

Objetivo: Analizar la incidencia de las complicaciones urológicas, fístulas y estenosis urinarias, observadas en nuestra serie de 282 trasplantes así como el tratamiento de las mismas.

Material y métodos: Entre diciembre de 1995 y octubre de 2005, hemos realizado 282 trasplantes renales. La complicación urológica más frecuente ha sido la fistula urinaria observándose en 24 casos $(8,5 \%)$, seguida de la estenosis ureteral en 18 casos $(6,4 \%)$ y en 5 casos se observaron ambas complicaciones. Dentro de los datos recogidos de los pacientes destacamos el tiempo de diagnóstico, las técnicas de imagen empleadas, el tipo de catéter ureteral empleado y la evolución clínica.

Resultados: El tratamiento endourológico mediante nefrostomía percutánea, catéter doble $\mathrm{J}$ y endoprótesis ha sido eficaz en el 76,4\% de las fístulas urinarias, en el 66,7\% de las estenosis ureterales y en el $60 \%$ de los pacientes que presentaron ambas complicaciones.

Conclusión: El tratamiento endourológico ha reemplazado a las técnicas de cirugía abierta en el manejo de la mayoría de las estenosis ureterales y fístulas urinarias post-trasplante renal, al ser un tratamiento definitivo con una baja morbilidad asociada.

Palabras clave: Trasplante renal. Complicaciones urológicas. Catéter.

\section{ABSTRACT}

\section{DIAGNOSIS AND TREATMENT OF UROLOGICAL COMPLICATIONS IN KIDNEY TRANSPLANTS}

Objective: To analyze the incidence of urological complications, like fistula and stenosis in our series of 282 renal transplants and their management.

Materials and methods: Between December 1995 and October 2005, 282 adult recipients underwent renal transplant .The most common urological complication was urinary fistula. This complication was observed in 24 cases (8.5\%), ureteral stenosis in 18 cases $(6.4 \%)$ and both of them in 5 (1.7\%). The items recorded on these patients included the time to diagnosis, the image technique, the type of ureteral stents and the clinical evolution.

Results: Endourologic treatment with percutaneos nefrostomy, double-J catheter and metalic endoprotesis was performed successfully in $76.4 \%$ of urinary fistula, in $66.7 \%$ of ureteral obstruction and in $60 \%$ of patients who developed both of them.

Conclusion: Endourologic procedures have replaced open reconstructive surgery in most patients with ureteral obstruction or urinary fistula after renal transplant, because they may offer a definitive treatment with low morbidity. 
El porcentaje de las complicaciones urológicas del trasplante renal ha ido variando a lo largo de los años debido a la prevención, diagnóstico y tratamiento precoz de las mismas ${ }^{1}$, destacando que la técnica quirúrgica del trasplante es la misma que la que se realizaba ya en el año $1960^{2}$.

En las series publicadas en los años setenta la tasa de complicaciones post-trasplante oscilaba entre un 3,5 y un $30 \%{ }^{3-6}$, mientras en las series recientes esta cifra se encuentra entre un 3 y un $12 \%^{7-22}$.

Sin embargo actualmente se esta observando un nuevo incremento tanto de las estenosis como de las fistulas urinarias, creemos que en relación con el uso de inmunosupresores como el Tacrolimus.

Estas complicaciones se producen como consecuencia de la alteración de la vascularización ureteral que acontece durante la manipulación del injerto en la extracción y posterior trasplante, así como con la reimplantación ureterovesical realizada. Este compromiso vascular genera un daño isquémico y consecuentemente una necrosis que afecta con mayor frecuencia al tercio distal del uréter ${ }^{1}$, pudiendo llevar a la pérdida del injerto en un 10 a un $15 \%$ de los casos ${ }^{1}$.

Tradicionalmente el tratamiento de estas alteraciones ha sido la cirugía abierta mediante técnicas de reimplantación ureteral ${ }^{1,3,18,19,23,24}$.

Sin embargo, desde el desarrollo de las técnicas endourológicas en la década de los ochenta el manejo de las fístulas y las estenosis ureterales por estos procedimientos está siendo una alternativa eficaz.

En 1978 se describe por primera vez las ventajas del empleo de un catéter de nefrostomía percutánea en un riñón trasplantado obstruido ${ }^{25}$.

De acuerdo con ésto algunos grupos de trasplante renal están realizando una cateterización sistemática de la anastomosis vésico-ureteral, con el fin de disminuir la tasa de complicaciones quirúrgicas ${ }^{21,22,26-29}$.

Tras realizar una revisión de la literatura sobre el manejo de estas complicaciones hemos visto que no existen unas pautas establecidas.

En el presente trabajo analizamos de manera retrospectiva el porcentaje de estenosis y fístulas urinarias en nuestra serie de trasplantes renales, estudiando el intervalo de aparición, el diagnóstico y el tratamiento de las mismas.

\section{MATERIAL Y MÉTODOS}

En el periodo comprendido entre diciembre de 1995 y octubre de 2005 hemos realizado 282 trasplantes renales.

Mediante la realización de un estudio retrospectivo hemos detectado 47 casos $(16,6 \%)$ de complicaciones urológicas. De ellos 24 (8,5\%) fueron fístulas urinarias, $18(6,4 \%)$ presentaron estenosis ureterales y en $5(1,7 \%)$ se observaron ambas alteraciones.

La media de edad ha sido de 53 años (26-73), siendo la mayoría de los receptores varones 29 (62\%).

Todos los riñones procedían de donante cadáver. En $44(93,6 \%)$ casos el trasplante se realizó en la fosa iliaca derecha y sólo en $3(6,4 \%)$ en la fosa iliaca izquierda, dos de ellos por existir importantes calcificaciones vasculares en la fosa iliaca derecha y en el caso restante por tratarse de un segundo trasplante. De los 47 injertos, 27 (57\%) fueron riñones derechos y 20 (43\%) riñones izquierdos.

Todas las anastomosis vasculares fueron término-laterales a los vasos iliacos externos y la continuidad de la vía urinaria se estableció en todos los casos realizando una ureteroneocistostomía mediante la técnica intravesical de Paquin. Sólo en 4 pacientes se colocó de forma profiláctica un catéter doble $\mathrm{J} 6 \mathrm{Ch}$.

Las estenosis ureterales se sospecharon por la elevación de las cifras de creatinina y urea observadas en los estudios analíticos realizados de control.

En la fístula urinaria se produjo un aumento del débito a través del catéter de drenaje ó a través de la herida quirúrgica en aquellos pacientes en los que ya se había retirado el tubo de drenaje. El análisis bioquímico del líquido recogido demostró que se trataba de orina.

La realización de una ecografía del injerto evidenció distintos grados de hidronefrosis y la cistografía descartó o confirmó la presencia de una fístula vesical.

Posteriormente realizamos una pielografía anterógrada dirigida mediante ecografía y fluoroscopia convencional, observando el tipo de alteración y su localización a lo largo de la vía urinaria. De esta forma diagnosticamos 24 casos de fístulas urinarias, 18 de estenosis ureterales y 5 en los que existían ambas alteraciones (Fig. 1). 


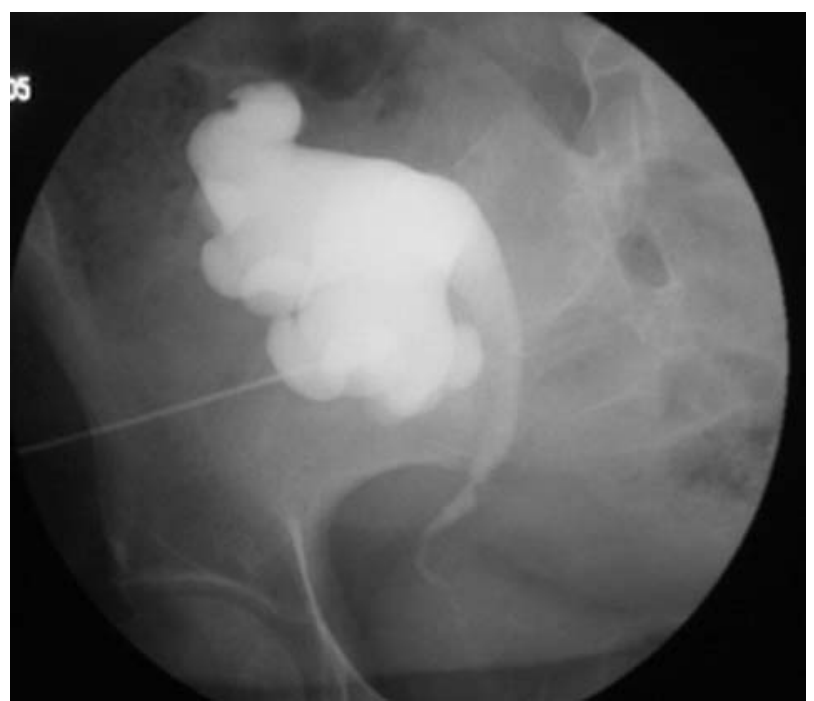

FIGURA 1: Pielografia percutánea que muestra una estenosis en el tercio distal del uréter en un paciente trasplantado renal.

De los 24 casos de fístulas urinarias, 3 $(12,5 \%)$ fueron caliciales, $3(12,5 \%)$ se localizaron en la unión pieloureteral, 14 (58,4\%) en el tercio distal del uréter próximas a la reimplantación urétero-vesical y los 4 (16,7\%) restantes fueron fístulas vesicales.

En lo que respecta a las estenosis de la vía urinaria de los 18 casos, $6(33,4 \%)$ se localizaron en el uréter proximal cerca de la unión pieloureteral, $3(16,6 \%)$ en el tercio medio del uréter, y en 9 (50\%) la estenosis se localizó en el uréter distal.

De los 5 pacientes que presentaron ambas alteraciones, en 4 (80\%) la estenosis y la fístula se localizaron en el tercio distal del uréter, mientras que sólo en 1 caso la estenosis fue a nivel del uréter proximal y la fístula asociada en el tercio distal del mismo.

El tiempo medio de aparición desde la realización del implante renal en el caso de las fístulas ureterales fue de 9,6 (1-35) días y en las estenosis de 176 (2-730) días, destacando cuatro casos de estenosis que aparecieron 2 años después de la realización del trasplante renal.

\section{RESULTADOS}

Las fístulas vesicales se trataron en 3 de los 4 $(75 \%)$ casos manteniendo la sonda vesical durante una media de 44 (33-60) días. Solamente en un paciente, en el que la fístula vesical apareció muy precozmente tras la cirugía, se reconstruyó la anastomosis vesical quirúrgicamente.

Los 3 casos de fístulas caliciales requirieron la colocación de una nefrostomía percutánea más un catéter doble $\mathrm{J}$ durante 45 días con resolución del 100\% de las mismas (Tabla 1).

Tabla 1

Tratamiento de las complicaciones

\begin{tabular}{|c|c|c|c|c|}
\hline Casos & Complicacion & Tratamiento & Eficaz & $\begin{array}{l}\text { Tratamiento } \\
\text { complementario }\end{array}$ \\
\hline 3 & Fístula calicial & Catéter Doble J 45 días & Si $(100 \%)$ & No \\
\hline 4 & Fistula vesical & Sonda Vesical 44 días & $\mathrm{Si}(75 \%)$ & $25 \%$ Cirugía abierta \\
\hline 17 & Fistula ureteral & $\begin{array}{l}\text { Nefrostomía, Catéter } \\
\text { Doble J } 58 \text { días }\end{array}$ & Si $(58,8 \%)$ & $\begin{array}{l}\text { 17,4\% desarrollan estenosis, } \\
\text { (Doble J / Endoprótesis) } \\
23,6 \% \text { Cirugía abierta } \\
\text { (nefrectomía derecha- anastomosis } \\
\text { pielo-piélica) }\end{array}$ \\
\hline 18 & Estenosis ureteral & Catéter Doble J 70 días & $\mathrm{Si}(50 \%)$ & $\begin{array}{l}\text { 5,5\% Meatotomía } \\
\text { 22,2\% Endoprótesis } \\
\text { 22,2\% Cirugía Abierta } \\
\text { (nefrectomía derecha- } \\
\text { anastomosis pielo-piélica) }\end{array}$ \\
\hline 5 & $\begin{array}{l}\text { Fistula y estenosis } \\
\text { ureterales }\end{array}$ & Catéter Doble J 70 días & $\mathrm{Si}(60 \%)$ & $\begin{array}{l}40 \% \text { Cirugía Abierta } \\
\text { (nefrectomía derecha- } \\
\text { anastomosis pielo-piélica) }\end{array}$ \\
\hline
\end{tabular}


Los 17 casos de fístulas ureterales, 3 a nivel de la unión pieloureteral y 14 en el tercio distal del uréter, fueron tratados inicialmente mediante técnicas endourológicas. Se colocó una nefrostomía percutánea y un catéter doble $\mathrm{J}$ anterógrado que permaneció durante una media de 58 días (30-150), siendo eficaz en 10 casos (58,8\%). En 3 $(17,6 \%)$ tras la retirada del catéter doble $\mathrm{J}$ se observa la aparición de una estenosis ureteral distal. En uno de estos casos se realiza una dilatación con catéter-balón más la colocación de un catéter doble $\mathrm{J}$ durante un mes más solucionándose la estenosis ureteral. En los otros 2 casos se decidió colocar una endoprótesis ureteral tipo VIABAHN de $5 \mathrm{~cm}$ con $7 \mathrm{~mm}$ de diámetro. Estas prótesis son flexibles con un soporte externo de nitinol y un revestimiento de politetrafluoroetileno expandido, impermeable y no adherente a la mucosa lo que permite su extracción previa irrigación con agua fría (Fig. 2).

La evolución posterior de estos pacientes a medio plazo (12 meses) se realizó mediante ecografía y urografia. En uno de los casos se ha observado una permeabilidad completa del uréter y una ausencia de ectasia pieloureteral. En el otro paciente se confirmó el desplazamiento de la prótesis hacia la unión pieloureteral persistiendo

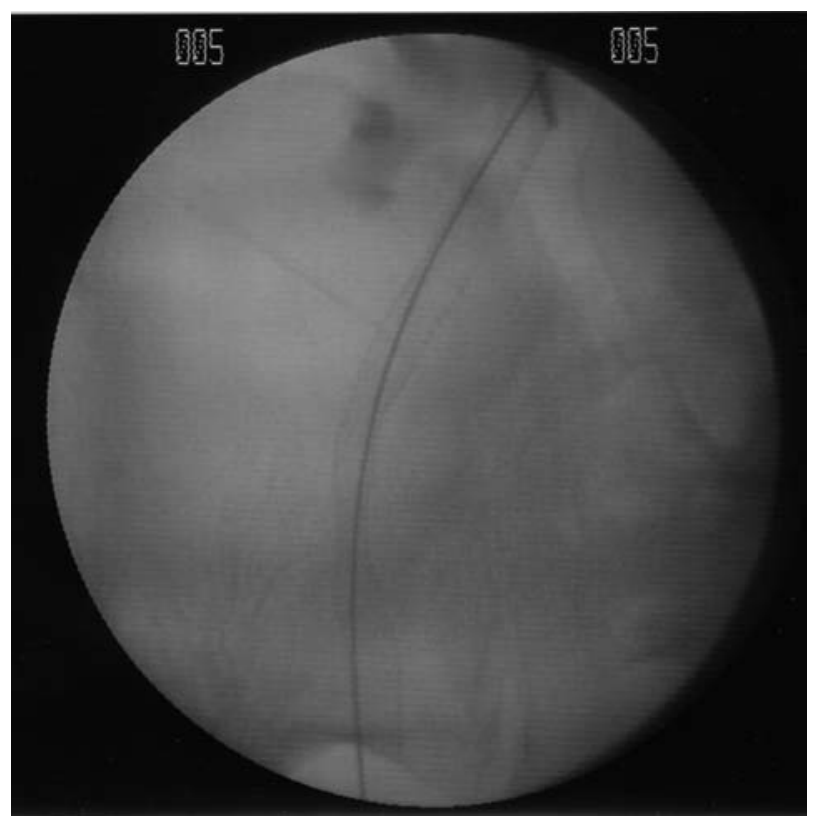

FIGURA 2: Pielografia ascendente: Estenosis en el tercio medio del uréter. Se observa la colocación de una Endoprótesis parcialmente abierta en el interior del uréter. la estenosis ureteral distal al injerto metálico. Se procedió a la retirada de la prótesis de forma anterógrada a través de un catéter de nefrostomía comprobándose la persistencia de la estenosis y colocándose una nueva endoprótesis de mayor longitud.

De esta forma 13 de los $17(76,4 \%)$ casos de fístulas ureterales fueron resueltos mediante técnicas endourológicas. En los 4 pacientes restantes $(23,6 \%)$ la fístula ureteral persistió a pesar de la colocación de un catéter doble J, por lo que se optó por la cirugía abierta realizándose una anastomosis pielopiélica (Tabla 2).

Tabla 2

Eficacia de las técnicas endourológicas

\begin{tabular}{lcc}
\hline Complicación & $\begin{array}{c}\text { Tratamiento } \\
\text { endourológico } \\
\text { (Nefrostomia, Doble J, } \\
\text { Endoprótesis) }\end{array}$ & $\begin{array}{c}\text { Cirugia } \\
\text { abierta }\end{array}$ \\
\hline Fistula Ureteral & $76,4 \%$ & $23,6 \%$ \\
Estenosis Ureteral & $66,7 \%$ & $33,3 \%$ \\
Fistula + Estenosis Ureteral & $60 \%$ & $40 \%$ \\
\hline
\end{tabular}

La estenosis de la vía urinaria fue observada en 18 pacientes trasplantados $(6,4 \%)$. En todos los pacientes se realizó un tratamiento mediante técnicas endourológicas. Se colocó una nefrostomía percutánea seguido de un catéter doble J. En dos casos fue necesaria previamente la dilatación del trayecto ureteral mediante un catéter-balón. La media de permanencia del catéter doble $J$ fue de 70 días (30-150), siendo este tratamiento eficaz en 9 pacientes (50\%). En un caso tras la retirada del catéter fue necesaria la realización de una meatotomía ureteral (Fig. 3).

En cuatro pacientes $(22,2 \%)$ se observó la persistencia de la estenosis por lo que se coloca una endoprótesis ureteral tipo VIABAHN $5 \mathrm{~cm}$ con 7 $\mathrm{mm}$ de diámetro, salvo en un caso en el que se colocaron dos prótesis telescopadas por la gran longitud de la estenosis. El seguimiento de estos pacientes ha sido de 12 meses (5-24). Realizamos controles analíticos y estudios de imagen mediante ecografía y urografía. Dos pacientes (50\%) mostraron una permeabilidad completa del uréter. En un tercer paciente se detectó una ectasia pielocalicial por migración de la prótesis hacia el extremo distal de uréter. La alteración fue tratada median- 


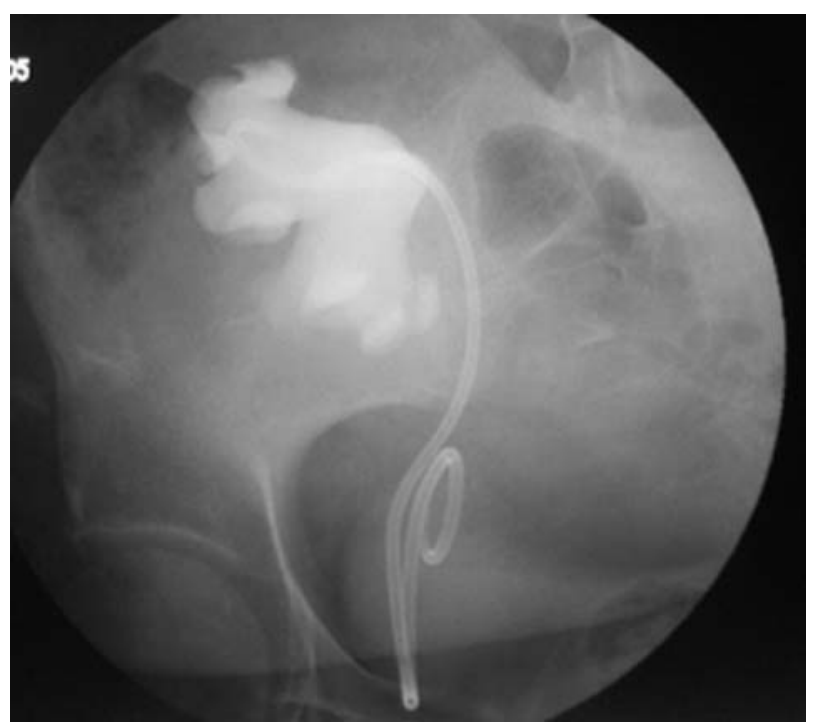

FIGURA 3: Colocación de catéter Doble $J$ en paciente trasplantado renal que presenta una estenosis ureteral distal.

te la retirada de la prótesis y la colocación de una nueva endoprótesis tipo VIABAHN pero de mayor longitud ( $8 \mathrm{~cm}$ por $8 \mathrm{~mm}$ de diámetro).

En el caso restante se produjo una obstrucción de la prótesis por hiperplasia urotelial. En un primer momento se intentó la colocación de un catéter doble $\mathrm{J}$ a través de la prótesis para restablecer la permeabilidad del uréter, no siendo posible por lo que se realizó cirugía abierta consistente en una anastomosis pielopiélica.

En otros 4 casos $(22,2 \%)$ el tratamiento endourológico no fue eficaz siendo necesaria igualmente una anastomosis pielopiélica.

De esta manera el tratamiento de las estenosis ureterales mediante técnicas endourológicas, colocando un catéter doble $\mathrm{J}$ ó una endoprótesis ureteral fue eficaz en el $66,7 \%$ de los casos, realizándose cirugía abierta en el 33.3\% restante de los casos (Tabla 2).

En 5 casos $(1,7 \%)$ se observó la presencia de una fistula urinaria asociada a estenosis ureteral. En 4 pacientes ambas alteraciones se localizaron a nivel del uréter distal, mientras que sólo en 1 caso la estenosis fue a nivel del uréter proximal y la fístula asociada en el tercio distal del mismo. En 3 pacientes (60\%) las alteraciones fueron tratadas eficazmente mediante la colocación de nefrostomía percutánea más catéter doble $\mathrm{J}$ durante 70 días (30-120). En los dos casos restantes (40\%) no fue posible la colocación de un catéter doble $\mathrm{J}$ debido a la importante estenosis que presentaba por lo que se realizó una anastomosis pielopiélica.

\section{DISCUSIÓN}

Las complicaciones urológicas representan la principal causa de morbimortalidad del enfermo trasplantado. La más frecuente sigue siendo la estenosis ureteral seguida de la fistula urinaria ${ }^{17,30}$. Sin embargo en nuestra serie de trasplantes renales hemos observado un mayor número de casos de fístulas urinarias $(8,5 \%)$.

Tradicionalmente el tratamiento de estas complicaciones ha sido la cirugía abierta, sin embargo estudios realizados posteriormente destacando autores como Barbaric y Thomson han puesto de manifiesto que el empleo de catéteres de derivación urinaria disminuyen el porcentaje de complicaciones y con ello la morbimortalidad de los pacientes trasplantados ${ }^{19,31,32}$.

En nuestra serie de trasplante renal el 76,4\% de las fistulas ureterales fueron tratados mediante técnicas endourológicas. En el 23,6\% restante persistió la fístula ureteral por lo que se optó por la cirugía abierta realizándose anastomosis pielopiélica.

Al igual que nosotros, diversos autores ${ }^{1,33}$ defienden el empleo inicialmente de técnicas endourológicas para el manejo de las fístulas de la vía urinaria por su alta tasa de éxito recurriendo a la cirugía abierta cuando este primer escalón terapéutico no resulta eficaz.

Shoskes et al. refieren una tasa de estenosis del 3,6\%, y Butterrworth et al. obtienen una frecuencia del $2,8 \%$.

Nosotros hemos encontrado un $6,4 \%$ de estenosis de la vía urinaria constituyendo nuestra segunda complicación urológica más frecuente.

En todos los pacientes se comenzó con un tratamiento mediante técnicas endourológicas siendo eficaz en el $66,7 \%$ de los casos, realizándose cirugía abierta en un 33,3\%.

Resultados semejantes quedan recogidos en la literatura y así Li Marzi et al. ${ }^{1}$ en una serie de 453 trasplantes obtienen una tasa de estenosis del 5,6\%, siendo el tratamiento endourológico eficaz en el 58\% de los casos y realizando cirugía abierta tras el fracaso de estas técnicas. 
Whang et al. ${ }^{33}$ en su serie de 1.066 trasplantes renales encuentran una frecuencia de estenosis del 2,7\%.y una tasa de éxito con el tratamiento endourológico del 85\%. Estos autores destacan además que uno de los principales factores que contribuye a la aparición de las estenosis es la longitud del uréter siendo beneficioso el empleo de un segmento corto del mismo.

Pappas et al. ${ }^{23}$ en su serie de 932 implantes renales con una tasa de estenosis urinaria del $4,7 \%$, establece el empleo de técnicas endourológicas como tratamiento inicial y definitivo por los buenos resultados que ofrece (éxito $84,6 \%$ ) y la baja morbimortalidad que conlleva.

\section{CONCLUSIONES}

- El factor fundamental en la aparición de las fístulas y estenosis de la vía urinaria es la "vascularización“ del uréter. Por ello debemos ser muy rigurosos en la realización de la extracción y posterior implante.

- Una vez que se producen estas complicaciones urológicas es el diagnóstico y el tratamiento precoz de las mismas lo que nos permitirá evitar la pérdida del injerto. La cirugía abierta ha sido hasta hace pocos años el tratamiento de elección de estas complicaciones.

- Las técnicas endourológicas permiten resolver de forma rápida una obstrucción renal aguda, diagnosticar la alteración que motiva dicha obstrucción y realizar un tratamiento mediante la colocación de un catéter doble $\mathrm{J}$ o una endoprótesis.

- Nuestro trabajo demuestra que las técnicas endourológicas constituyen un tratamiento no sólo inicial sino también definitivo de las complicaciones urológicas $(76,4 \%$ de las fístulas ureterales y $66,7 \%$ de las estenosis urinarias) más frecuentes del trasplante renal.

\section{REFERENCIAS}

1. Li Marzi V, Filocamo MT, Dattolo E, Zanazzi M, Paoletti MC, Marzocco M et al. The treatment of fistulae and ureteral stenosis after kidney transplant. Transplant Proceedings 2005;37(6):2516-2517.

2. Malek GH, Uehling DT, Danouk A. Urological complications of renal transplantation. J Urol. 1973;109(2):173-176.

3. Gil Martínez P, Liédana Torres JM, Rocales Badal A. Análisis de nuestra serie de trasplantes renales, complicaciones urológicas y supervivencia. Actas Urol Esp 1998; 22(4):326-335.
14. Martín García B, Correas MA, Portillo JA, Gutiérrez JL, Hernández R. Trasplante renal. Tratado de Urología (Jiménez Cruz y Rioja Sanz Editores). p: 1763. Ed. J.R. Prous Editores. Barcelona, 1993.

15. Marx W1, Halasz N, Mclaughlin AP. Urological complications in renal transplant. J Urol 1974; 112: 561.

16. Salvatierra O, Olcott JR; Amend WY. Urological complications of renal transplantation can be prevented or controlled. J Urol. 1977Apr; 117(4):421-424.

17. Cagatay Gogus, Onder Yaman, Tarkan Soygür, Yasar Bedük, Orhan Gogus. Urological complications in renal transplantation: Long-term follow-up of the Wooddruff ureteroneocystostomy procedure in 433 patients. Urol Int 2002;69:99-101.

18. Kashi SH, Lodge JP, Giles GR, Inwing HC. Ureteric complications of renal transplantation. Br J Radiol 1992;70: 139-143.

19. Gibbons WS, Barry JM, Hefty TR. Complications following unstented parallel incision extravesical ureteroneocystostomy in 1000 kidney transplants. J Urol 1992;148(1):3840.

10. Shoskes DA, Hamburg D, Cranston D, Morris PJ. Urological complications in 1000 consecutive renal transplants recipients. J Urol. 1995;153(1):18-21.

11. Sagalowsky AI, Ransler CW, Peters PC, Dickermon RM, Gailiunas P. Urologic complications in 505 renal transplants with early catheter removal. J Urol. 1983;129(5): 929-932.

12. Mundy AR, Podesta ML, Bewich M, Rudge CJ, Ellis FG. The urological complications of 1000 renal transplants. $\mathrm{Br}$ J Urol. 1981;53(5):397-402.

13. Cimic J, Meuleman EJH, Oosterhof GON, Hoitsma AJ. Urological complications in renal transplantation. A comparison between living-related and cadaveric grafts. Eur Urol. 1997;31(4):433-435.

14. Benoit G, Blanchet P, Moukarzel M, Hiesse C, Bensadoun $\mathrm{H}$, Bellamy $\mathrm{J}$ et al. Surgical complications in kidney transplantation. Transplant Proc 1994;26(1):287-288.

15. Kinnaert P, Hall M, Janssen F, Vereerstraeten P, Toussaint C, Van Geertruyden J. Ureteral estenosis after kidney trasplantation: true incidence and long-term follow-up after surgical correction. J Urol. 1985;133(1)17-20.

16. Loughlin KR, Tilney NL, Rihie JP. Urological complications in 718 transplant patients. Surgery 1984;95(3):297-302.

17. Mundy AR, Podesta ML, Bewich M, Rudge CJ, Ellis FG. The urological complications of 1000 renal transplants. Br J Urol. 1981;53(5):397-402.

18. Pozo B, Burgos FJ, Linares A, Briones G, Pascual J, Marcen $\mathrm{R}$ et al. Estenosis ureteral post-trasplante renal: Tratamiento con prótesis metálica autoexpandible. Actas Urol Esp. 2003; 27(3):190-195.

19. Briones G, Burgos FJ, Pascual J, Marcen R, Pozo B, Arambarri A et al. Estudio comparativo entre anastomosis ureteral con y sin cateterización con doble $\mathrm{J}$ en trasplante renal. Actas Urol Esp. 2001;25(7):499- 503.

20. Rigg KM, Proud G, Taylor RM. Urological complications following renal transplant. A study of 1016 consecutive transplants from a single center. Transplant Int 1994;7 (2): 120-126.

21. Kumar A, Kumar R, Bhandari M. Significance of routine JJ stenting in living related renal transplantation: a prospective randomised study. Transplant Proc. 1998;30(7):29952997. 
22. Kumar A, Verma B, Srivastava A, Bhandari M, Gupta A, Sharma R. Evaluation of the urological complications of living related renal transplantation at a single center during the last 10 years: Impact of the double-J stent. $\mathrm{J}$ Urol. 2000; 164(3Pt1):657-660.

23. Pappas P, Stravodimos KG, Adamakis I, Leonadou P, Zavos $\mathrm{G}$, Constantinides $\mathrm{C}$ et al. Prolonged ureteral stenting in obstruction after renal transplantation: Long-term results. Transplant Proc. 2004;36(5):1398- 1401.

24. Alcaraz A, Bujons A, Pascual X, Juaneda B, Marti J, de la Torre $\mathrm{P}$ et al. Percutaneous management of transplant ureteral fistulae is feasible in selected cases. Transplant Proc. 2005;37(5):2111-2114.

25. Barbaric ZL, Thomson KR. Percutaneos nephrostomy in the management of obstructed renal transplants. J Urol. 1978; 124:781.

26. Benoit G, Lassal M, Blanchet P, Bellamy J, Charpentier B, Jardin A. The treatment or urinary fistula in renal transplantation. Prog Urol. 1994;4(4):501-508.

27. Nicol DL. Routine use of indwelling ureteral stents in renal transplantation. J Urol. 1993;150(5Pt1)1375-1379.

28. Kumar A, Srinath ES, Gulati S, et al. Conservative and endourological management of urological complications in renal transplantation. Ind J Urol 1995;11:76.
29. Koo Seen Lin LC, Bewick M, Koffman CG. Primary use double $\mathrm{J}$ silicone ureteric stent in renal transplant. $\mathrm{Br} \mathrm{J}$ Urol 1993; Suppl.72(5pt2):697-701.

30. Starzl TE, Groth CG, Putnaun CW, Penn I, Halgrimson CG, Flatmark A et al. Urological complications in 216 human recipients of renal transplants. Ann Surg. 1970;172(1):1-22.

31. Golstein I, Cho SI, Olsson CA. Nephrostomy drainage for renal transplant complications. J Urol. 1981; 126(2):159-163.

32. Campbell SC, Streem SB, Zelch M, Hodge E, Novick AC. Percutaneous management of transplant ureteral fistulas: patient selection and long-term results. J Urol. 1993;150 (4):1115-1117.

33. Whang M, Geffner S, Baimeedi S, Bonomini L, Mulgaonkar S.: Urologic complications in over 1000 kidney transplants performed at the Saint Barnabas Healthcare System. Transplant Proc. 2003;35(4): 1375-1377.

Dra. V. Gonzalo Rodríguez

E-mail: VictoriaCGR@hotmail.com

(Trabajo recibido el 6 de febrero de 2006) 